\title{
Biomarkers in immunotherapy: literature review and future directions
}

\author{
Rebecca Pharaon ${ }^{1}$, Maria A. Koczywas ${ }^{2}$, Sabrina Salgia ${ }^{1}$, Atish Mohanty ${ }^{1}$, Erminia Massarelli ${ }^{1}$ \\ ${ }^{1}$ Department of Medical Oncology and Therapeutics Research, City of Hope National Medical Center, Duarte, CA, USA; ${ }^{2}$ Wroclaw Medical \\ University, Wybrzeze L. Pasteura 1, 50-367 Wroclaw, Poland \\ Contributions: (I) Conception and design: R Pharaon, M Koczywas, S Salgia, E Massarelli; (II) Administrative support: R Pharaon, E Massarelli; \\ (III) Provision of study materials or patients: R Pharaon, M Koczywas, S Salgia, E Massarelli; (IV) Collection and assembly of data: R Pharaon, A \\ Mohanty, E Massarelli; (V) Data analysis and interpretation: All authors; (VI) Manuscript writing: All authors; (VII) Final approval of manuscript: All \\ authors. \\ Correspondence to: Dr. Erminia Massarelli. City of Hope National Medical Center, 1500 W Duarte Road, Duarte, California, 91010 , USA. \\ Email: emassarelli@coh.org.
}

\begin{abstract}
Within the past decade, immunotherapy has revolutionized the treatment of advanced nonsmall lung cancer (NSCLC). Immune checkpoint inhibitors (ICIs) such as pembrolizumab, nivolumab, atezolizumab, and durvalumab have shown superiority over chemotherapy regimens in patients with programmed death-ligand 1 (PD-L1) expression. Several predictive molecular biomarkers, including PDL1 expression and high tumor mutation burden, have shown utility in discovering lung cancer patient groups that would benefit from ICIs. However, there remains to be a reliable imaging biomarker that would clearly select patients, through baseline or restaging imaging, who would respond or have a prolonged response to ICIs. The purpose of this review is to highlight the role of ICIs in patients with advanced NSCLC and past or current studies in potential biomarkers as well as future directions on the role of imaging in immunotherapy.
\end{abstract}

Keywords: Non-small cell lung cancer (NSCLC); immunotherapy; molecular biomarkers; in vivo imaging; imaging biomarkers

Submitted Dec 28, 2019. Accepted for publication Mar 31, 2020.

doi: $10.21037 /$ jtd.2020.04.15

View this article at: http://dx.doi.org/10.21037/jtd.2020.04.15

\section{Introduction}

Immunotherapy has revolutionized the treatment of patients diagnosed with non-small cell lung cancer (NSCLC), a cancer estimated to cause the most cancer deaths in the United States in 2019 in both males and females (1). Introduction of anti-programmed cell death protein 1 (PD-1) and anti-programmed death-ligand 1 (PD-L1) monoclonal antibodies such as pembrolizumab, nivolumab, atezolizumab, and durvalumab in the field of lung cancer showed efficacy of these drugs in terms of overall survival and progression free survival over or in addition to traditional chemotherapy (2-6). Selection of patients who will benefit is crucial to maximize response to treatment and limit treatment toxicities associated with immunotherapy (7).

Currently, to predict if patients will benefit from immune checkpoints inhibitors (ICIs), known molecular biomarkers such as PD-L1 and tumor mutation burden (TMB) are determined via immunohistochemical (IHC) assays or next-generation sequencing (NGS) testing. These biomarkers have shown efficacy in selecting patients to undergo treatment with immunotherapy over traditional cytotoxic chemotherapy treatment. A landmark Phase I trial demonstrated prolonged progression-free survival (PFS) with nivolumab and ipilimumab in patients with high tumor mutation burden regardless of PD-L1 expression (8), and most recently, results from a Phase III trial confirmed efficacy of this combination as first-line treatment in 


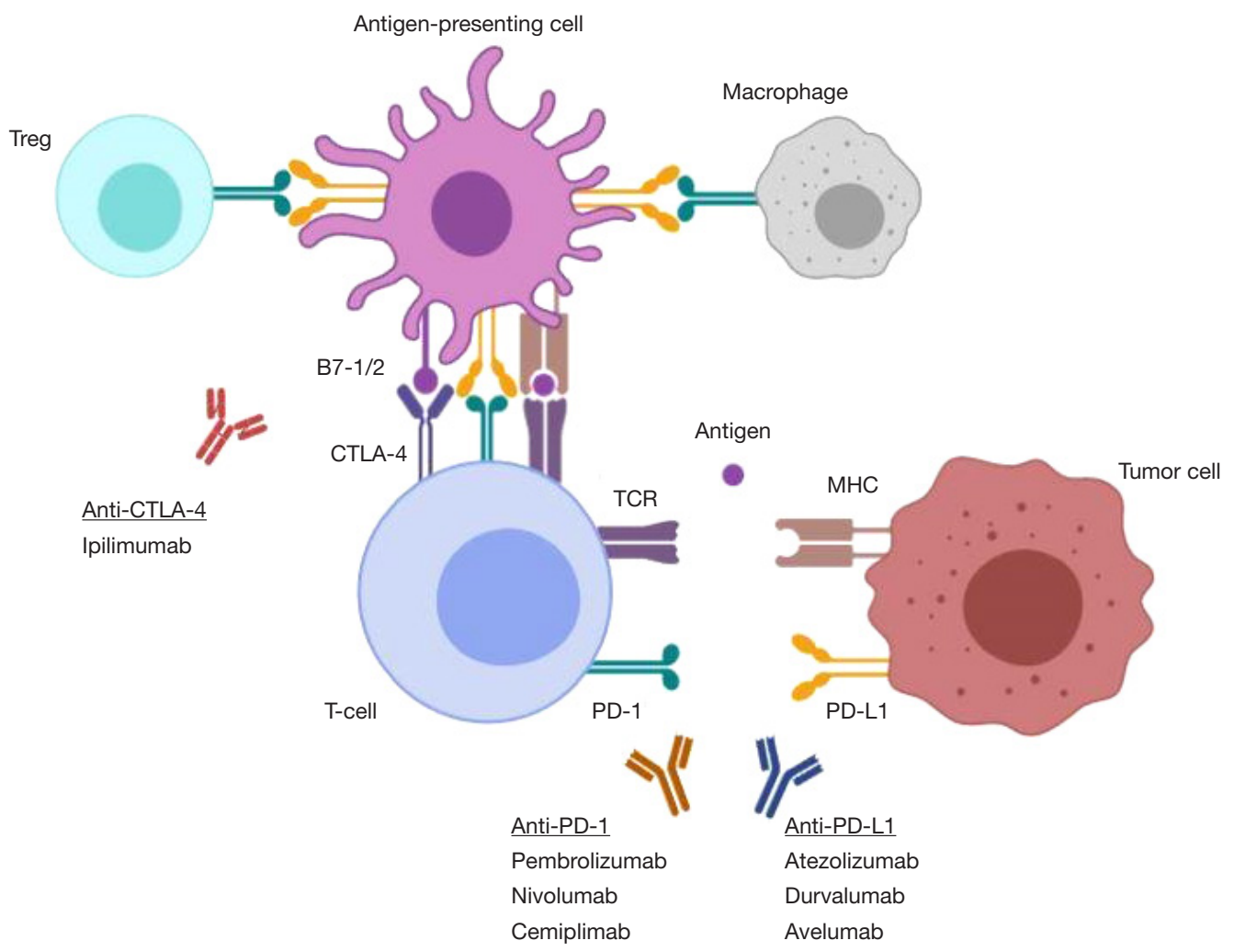

Figure 1 Anti-PD-1, anti-PD-L1, and anti-CTLA-4 monoclonal antibodies targets and mechanisms.

NSCLC irrespective of any biomarker (9). Keynote-024 reported superior overall survival (OS) and PFS with monotherapy pembrolizumab in patients with high $\mathrm{PD}$ L1 expression [ $\geq 50 \%$ tumor proportion score (TPS)] (2). However, predicting initial or continued response to immunotherapy remains largely unknown and further research is necessary to develop reliable biomarkers.

A new field of investigating response to treatment has recently gained traction: using imaging to determine different biomarkers that can help guide treatment decisions for oncologists. Many ongoing trials are investigating imaging biomarkers that can be used in computed tomography (CT), magnetic resonance imaging (MRI), and positron emission tomography-computed tomography (PET/CT) scans including cluster of differentiation 8 (CD8) cells, chimeric antigen receptor-based $\mathrm{T}$ (CAR T) cells, and TMB. These noninvasive examinations would coincide with routine imaging and are better tolerated by patients compared to biopsy procedures or blood samples that are required at different timepoints to determine the evolving tumor molecular signature by NGS or IHC.
Visual detection of CD8 cells represents a good predictive indicator to estimate anti-tumor response; in fact, it helps scientists to better understand the dynamic of immune response on cancer growth and possible regression. Similarly, applying this idea in the field of CAR T-cells therapies is extremely beneficial as these cells could be exploited for repeated imaging. Without the capabilities of tracking specific cells that were used or targeted during therapy, it is near impossible to monitor and assess the efficacy or safety of the treatment.

This review focuses on established and promising molecular and imaging biomarkers that could help in selecting patient groups that would benefit from immunotherapy.

\section{Immune checkpoint inhibitors in NSCLC}

Immune checkpoints inhibitors (ICIs) work by blocking the natural inhibitory receptors/ligand interaction on immune cells (T-cells) and cancer cells to unleash immune response against cancer cells (Figure 1). The binding of PD-1, a 
T-cell inhibitory receptor, with PD-L1 leads to inhibition of T-cells function and allows tumor cell to escape from immune system response (Figure 1). By blocking PD-1/ PD-L1 interaction with anti-PD-1/PD-L1 monoclonal antibodies, the immune system can recognize tumor cells as foreign bodies. Most notably, ICIs have radically transformed treatment management of advanced NSCLC. Due to numerous landmark clinical trials, four agents have been approved by the Food and Drug Administration (FDA) as standard of care treatment of NSCLC: pembrolizumab, nivolumab, atezolizumab and durvalumab. Nivolumab, an anti-PD-1 monoclonal antibody, was first examined in NSCLC in comparison with standard chemotherapy and demonstrated improved overall survival in patients who had failed at least one prior line of chemotherapy $(10,11)$, establishing the potential significance of monotherapy immunotherapy in lung cancer. This concept was further proven with pembrolizumab which showed similar superior OS in NSCLC patients in second line (12). Later studies considered the effectiveness of ICIs as first-line treatment $(2,8,9)$, and for select patient groups with PDL1 TPS $\geq 50 \%$, pembrolizumab was approved as firstline standard of care for advanced NSCLC. There are many ongoing trials investigating ICI combinations with other immune-oncology (IO), incorporating immune and molecular biomarkers in NSCLC $(6,9,13)$. In this past year, long-term results from the Phase III Checkmate-017 and Checkmate-057 clinical trials were presented and demonstrated continued OS benefit of nivolumab in all cohort subgroups as a second-line treatment versus docetaxel (14). Although nivolumab was approved by the FDA in 2015 as a second-line therapy, these results show encouraging 4-year results even among patients who have a PD-L1 score $\leq 1 \%$. Recently, a Phase III trial of recurrent or metastatic NSCLC patients published results demonstrated a survival benefit of combining nivolumab and ipilimumab, a fully human anti-cytotoxic T-lymphocyte antigen 4 (CTLA-4) antibody, compared with chemotherapy as firstline treatment irrespective of PD-L1 expression (9).

\section{Molecular biomarkers of immunotherapy in NSCLC}

Testing PD-L1 expression using IHC has rapidly become a standardized test ordered at initial cancer diagnosis in addition to the advised NGS that includes established EGFR, ALK, ROS1, and BRAF de novo testing. Various antibodies have been studied to detect PD-L1 expression by IHC based on tumor histology and the use of the 22C3 anti-PD-L1 antibody has become standardized for PDL1 by IHC testing in NSCLC (15). The incorporation of PD-L1 testing into standard of care was established from several clinical trials indicating the strongest benefit of ICI in selected groups of patients expressing high PD-L1 (TPS $\geq 50 \%)(2,3)$. The estimated percentage of NSCLC patients who express PD-L1 ranges from $24 \%$ to $60 \%$ (16). Although it is unclear whether NSCLC patients with high PD-L1 expression have better prognosis than those with low or no levels of PD-L1, published data seems to suggest a poor prognosis associated with high PD-L1 levels (17-19). PD-L1 by IHC diagnostic has its disadvantages as it does not take into account tumor heterogeneity. Moreover, the dynamic nature of this marker causes changes in its expression levels in response to different factors, including radiation therapy, chemotherapy, wound formation, and the use of immune suppressing drugs. In fact, clinical benefit has been demonstrated in NSCLC patients whose tumors show low or no PD-L1 expression. However, despite its variability, as of today, $\mathrm{PD}-\mathrm{L} 1$ expression remains the best biomarker to predict response to immunotherapy thus far.

Another potential predictive marker of response to ICIs in NSCLC is TMB, which measures the average number of mutations carried by tumor cells. Tumors with high TMB can lead to more neoantigens in the body that are formed from mutations, resulting in a strong immune response to ICIs due to T-cells recognizing these neoantigens. Many studies have shown that patients with high TMB (greater or equal to 10 mutations per megabase) who undergo treatment with anti-PD-1/PD-L1 antibodies results in better PFS, objective response rate (ORR), and OS $(8,20,21)$. Limitations associated with TMB include: variability in TMB levels across different tumor types, inconsistent detection methods, and a lack of a standardized cutoff to define high TMB status. Recently, interest has risen in utilizing TMB in combination with PD-L1 expression to more specifically determine groups of patients who would respond to ICIs.

Human leukocyte antigen (HLA) is a complex of genes that encode the major histocompatibility complex (MHC) which regulates the immune system. Initially, tumor cells have a high level of class I MHC expression, which is the key for activating cytotoxic T-lymphocytes (CTLs). Over time, tumor cells that present with MHC-I are destroyed by CTLs and can transform to become MHC-I negative, thus making them less susceptible to CTLs destruction and immunological treatment (22). Loss of HLA class I 
expression has been reported in multiple cancers and studies have shown that expression of HLA-I on tumor cells is an important factor in evaluating immune infiltration (22). High expression of HLA-I is associated with high expression of PD-L1. In tumors with high expression of HLA-I and PD-L1, there exists a high intratumoral infiltration with CD8 cells. On the other hand, when the tumor is HLA-I negative, a significant reduction has been observed in the population of tumor infiltrating CD 8 cells. Since immunotherapy activates the immune system, including CD4/CD 8 cells and tumor-infiltrating lymphocytes, the importance of detecting HLA-I expression in patient tumors will also inform oncologists in estimating potential response to immunotherapy.

Various mutations and gene signatures in NSCLC have been reported to predict response to ICIs as well. The upregulation of interferon-gamma (IFN- $\gamma$ ), typically triggered by an immune stimulus, is a known marker of tumor response in different cancer types treated with immunotherapy, as described in several papers (23-25). A clinical trial in patients with NSCLC who received durvalumab demonstrated that a high IFN- $\gamma$ gene signature corresponded to better response rates and PFS (26). Multiple ongoing trials are examining therapies targeting IFN- $\gamma$ alone or in combination with ICIs in ovarian cancer, glioblastoma, and other solid tumors. On the other hand, studies have shown that specific mutations in NSCLC are associated with poor response to ICIs. In $K R A S$-mutated NSCLC, response to ICIs has been examined in various subgroups defined by co-mutations associated with KRAS. It was discovered that $K R A S$-mutated NSCLC tumors that also express $S T K 11$ or $L K B 1$ mutations cause primary resistance to anti-PD-L1 antibodies and predict poor outcomes (27). Numerous studies are currently underway to further classify patient subgroups who will respond or progress on ICIs.

\section{Immunotherapy response in imaging}

Response Evaluation Criteria in Solid Tumors (RECIST) is the standard criteria used to determine responses to therapy in clinical trials. This classification is based on the change in size of the tumor and is divided into four categories: complete response, partial response, stable disease, and progressive disease. However, RECIST can be insufficient in capturing pseudoprogression-a hallmark response in a subset of patients treated with antiPD-1/PD-L1 antibodies-and it may cause an incorrect response evaluation of immunotherapy. Psuedoprogression, a rare event which has been reported to occur in an estimated $2-5 \%$ of NSCLC cases (28-30), has been thought to be caused by the infiltration of immune cells causing an increase in the tumor which could mistakenly be attributed to growth due to progression of disease. Because of this phenomenon, three additional criteria were developed to provide a better assessment of the effect of immunotherapeutic agents: immune RECIST (iRECIST), immune-related RECIST (irRECIST), and immune-related response criteria (irRC). These criteria are typically used concurrently with RECIST to evaluate treatment response and any adverse events (AEs), especially immune-related $\mathrm{AEs}$ (irAEs). irAEs incidence rates vary in published clinical trials and retrospective analyses of patients treated with immunotherapy but are relatively common (7). General irAEs include colitis, thyroiditis, fatigue, and more, which are graded based on severity. Management of patients on immunotherapy requires medical oncologists and radiologists trained with the ability to recognize response clinical and radiologic response patterns of ICIs as well as adverse symptoms caused by ICIs.

\section{Imaging biomarkers of immunotherapy}

As immunotherapy became a significant therapeutic strategy across many cancer types, a new field of research opened to discover imaging biomarkers in addition to predictive molecular biomarkers. PET and single-photon emission computed tomography (SPECT) imaging utilize radioisotopes to label specific cells to target and visualize through imaging. Many clinical trials are currently underway to examine various imaging markers or radiolabels that could provide prognostic insight of response to antiPD-1/PD-L1 antibodies (Table 1).

Currently, detection of CD8 cells by any imaging technique is being investigated in various in vivo studies and clinical trials. CD8 cells belong to a larger group of cells called tumor infiltrating lymphocytes (TILs) which penetrate the tumor and its microenvironment to mediate immune response against tumor cells. CD8 cells have a significant role in eliminating tumor cells thus suggesting that high levels of CD8 cells can be a good prognostic marker of response in different cancer types $(31,32)$. Detection of CD8 through imaging could be helpful in not only assessing the usefulness of ICIs but to better visualize the influence of TILs on the tumor and its environment. A recent study by Seo et al. demonstrated 
Table 1 Ongoing clinical trials to predict response to immunotherapy in NSCLC using molecular imaging biomarkers

\begin{tabular}{|c|c|c|c|c|c|}
\hline Trial & Identifier & Status & Disease & Tracer & Phase \\
\hline $\begin{array}{l}\text { Imaging Tumor-infiltrating T-cells in } \\
\text { Non-small Cell Lung Cancer (Donan) }\end{array}$ & NCT03853187 & Recruiting & Non-small cell lung cancer & ${ }^{89} \mathrm{Zr}$-Durvalumab & Phase II \\
\hline $\begin{array}{l}{ }^{99 m} \text { Tc Labeled Anti-PD-L1 sdAb SPECT/ } \\
\text { CT in Assessment of PD-L1 Expression } \\
\text { in NSCLC }\end{array}$ & NCT02978196 & Recruiting & Non-small cell lung cancer & $\begin{array}{l}{ }^{99 m} \text { Tc-labeled anti-PD- } \\
\text { L1-sdAb }\end{array}$ & Phase I \\
\hline $\begin{array}{l}{ }^{18} \mathrm{~F}-\mathrm{PD}-\mathrm{L} 1 \mathrm{PET} / \mathrm{CT} \text { in Nivolumab } \\
\text { Treated Patients With NSCLC }\end{array}$ & NCT03564197 & Recruiting & $\begin{array}{c}\text { Stage IV non-small cell lung } \\
\text { cancer }\end{array}$ & ${ }^{18} \mathrm{~F}-\mathrm{PD}-\mathrm{L} 1$ & $\mathrm{~N} / \mathrm{A}$ \\
\hline MPDL3280A-imaging-IST-UMCG & NCT02453984 & Recruiting & $\begin{array}{l}\text { Locally advanced or } \\
\text { metastatic solid tumors }\end{array}$ & ${ }^{89} \mathrm{Zr}-\mathrm{MPDL}-3280 \mathrm{~A}$ & $\mathrm{~N} / \mathrm{A}$ \\
\hline
\end{tabular}

feasibility of tracking CD8 cells by PET imaging utilizing an anti-CD8 cys-diabody radiolabeled with ${ }^{64} \mathrm{Cu}$ in mouse models transplanted with an analogous form of HER2 breast cancer (33). Another study radiolabeled an antiCD8 cys-diabody with ${ }^{89} \mathrm{Zr}$ for non-invasive tracking of CD8 cells to visualize response, or lack of response, in syngeneic murine models via immuno-PET (34). The results demonstrated accurate binding of the anti-CD8 cys-diabody to CD8 T-cells and specific detection of CD8 T-cells by immuno-PET including after treatment with anti-CD137 antibody, a treatment that targets CD137 to augment activation of immune cells (34). Sun et al. studied a radiomic signature for $\mathrm{CD} 8$ cells in a retrospective cohort of patients using imaging data applied to different patient cohorts in the TCGA (35). The authors were able to use the CD8 cell radiomic signature to predict the tumor immune phenotype classifying tumors into 3 different types: immune inflamed, immune-excluded, or immunedesert. Generally, immune-inflamed types of tumors have the best chance to respond well to immunotherapy due to high CD8, immune cell infiltration, and PD-1/PD-L1 pathway activation $(36,37)$. This stresses the importance of visually tracking $\mathrm{CD} 8$ cells in vivo in patients undergoing treatment with immunotherapy to assess response and cell interaction with the tumor. Currently, there is an active Phase I/II clinical trial utilizing an anti-CD8 PET imaging agent, ZED88082A, radiolabeled with ${ }^{89} \mathrm{Zr}$ in solid tumor patients treated with anti-PD-1 or anti-PD-L1 alone or in combination with anti-CTLA-4 antibody ipilimumab. Another ongoing phase II clinical trial investigates the CD8 tracer, ${ }^{89} \mathrm{Zr}$-Df-IAB22M2C, in patients with metastatic solid tumors treated with standard of care monotherapy or combination ICIs (Table 1). Trials like these are comparing clinical response and immune infiltrates with uptake of radiolabeled CD8 tracers in PET/CT imaging before and after treatment with ICI.

Probes targeting other known immune markers have been investigated in preclinical settings and are currently undergoing clinical trials to determine efficacy and safety in human patients. Interleukin-2 (IL-2) receptors are expressed on activated T lymphocytes. Radiolabeled IL-2 has already shown efficacy for in vivo imaging of tumorinfiltrating CD25+ activated $\mathrm{T}$ lymphocyte to monitor CXCR4 antagonist therapy (38), a targeted therapy used for treatment of breast cancer. Anti-CTLA-4 monoclonal antibody ipilimumab has been proven to be effective in treating lung cancer when combined with nivolumab $(8,9)$. Results from a preclinical study have shown success in 
visualizing CTLA-4 on PET imaging through labeling with ${ }^{64} \mathrm{Cu}-1,4,7,10$-tetraazacyclododecane-N, $\mathrm{N}^{\prime}, \mathrm{N}^{\prime \prime}, \mathrm{N}^{\prime \prime \prime}$ tetraacetic acid-anti-mouse CTLA-4 mAb (64Cu-DOTAanti-CTLA-4 mAb) (39). PD-L1 probes have also been investigated in numerous preclinical studies in vitro and in murine mouse models (40-45). Moreover, multiple ongoing clinical trials are evaluating the efficacy of PET tracers with high affinity to PD-L1 such as ${ }^{99 \mathrm{~m}}$ Tc labeled anti-PD-L1 single domain antibody and ${ }^{18} \mathrm{~F}-\mathrm{PD}-\mathrm{L} 1$ (Table 1). Other active trials are radiolabeling a small dose of anti-PD-1/ PD-L1 agents such as avelumab, pembrolizumab, and durvalumab with ${ }^{89} \mathrm{Zr}$, a common radiolabel that attaches to monoclonal antibodies, in patients undergoing treatment with ICIs (Table 1) (46). Since PD-L1 expression has become an established molecular biomarker in tumor tissue, it is logical to replicate the results in imaging. If successful, this would reduce the need of invasive serial tissue biopsies that are currently used to consecutively map changes in mutational status throughout treatment. In the case for immunotherapy, oncologists would be able to longitudinally track PD-L1 expression based on probe activity on imaging.

CAR T-cell therapy has emerged within the past decade as a revolutionary treatment that genetically engineers a patient's T-cells to produce structures on their surface called chimeric antigen receptors (CARs) that are attuned to specific markers on tumor cells. These cells are then reinfused in the patient and are able to connect with proteins on tumor cells enabling recognition and killing of tumor cells, resulting in arming the patient's own immune system to respond against their cancer. Unfortunately, the difficulty in utilizing CAR T-cell therapy lies in identifying a target on tumor cells that is unique to them. CAR $\mathrm{T}$ therapies are approved by the Food and Drug Administration (FDA) in two cancer types-acute lymphoblastic leukemia in children (47) and advanced lymphoma in adults (48) —and both target CD19, a transmembrane glycoprotein that is expressed on the majority of $\mathrm{B}$ cell malignancies rendering it an ideal target. Aside from hematologic malignancies, this treatment so far has not shown equivalent success in solid tumors, with modest efficacy demonstrated in glioblastoma patients by targeting interleukin-13 receptor alpha 2 (IL-13 $\mathrm{R} \alpha 2)$ and other receptors that are specific to glioblastoma cancer cells $(49,50)$. Researchers are currently investigating whether imaging can be used to serially visualize response to CAR T-cells and recent data demonstrated preclinical efficacy of a prostate-specific membrane antigen-(PSMA-) targeting radiotracer to visualize CAR T-cells in NODSCID-Gamma mice using PET imaging $(51,52)$.

\section{Future directions}

Despite many advances in the field of cancer, specifically in NSCLC, oncologists still face many challenges in selecting patients who will derive benefit from ICIs and predicting tumor response. While a number of molecular biomarkers have been established as prognostic tools for immunotherapy response, they can be unreliable and further research is necessary in optimizing a selection of universal biomarker for immunotherapy. In addition, biomarker selection can help to lower the cost and toxicity of ineffective treatment. Preclinical data on radiolabeled probes is being published at rapid rates as numerous additional probes continue to be discovered. In vivo imaging can be a very useful non-invasive tool to assess the efficiency of immunotherapy and to predict potential resistance or toxicity of the treatment. Compared to biopsied tissue samples, it illuminates information about the heterogeneity of the entirety of tumors as well as the whole body, it is not restricted to the collected specimen, and it involves little to no risk for the patients. It can also be beneficial in dividing patients into two treatment groups of probable responders and likely non-responders. Furthermore, it can be an instrument to understand the mechanisms of action of different drugs, especially in immunotherapy considering that in vivo models have a very limited potential in studying the host-tumor heterogeneity. Therefore, it is very clear that molecular imaging as a non-invasive tool to predict and sequentially monitor response to immunotherapy has the potential to revolutionize cancer care and patient quality of life.

\section{Acknowledgments}

Funding: None.

\section{Footnote}

Provenance and Peer Review: This article was commissioned by the Guest Editor (Ammar Chaudhry) for the series "Role of Precision Imaging in Thoracic Disease" published in fournal of Thoracic Disease. The article was sent for external peer review organized by the Guest Editor and the editorial office.

Conflicts of Interest: All authors have completed the ICMJE uniform disclosure form (available at http://dx.doi. org/10.21037/jtd.2020.04.15). The series "Role of Precision Imaging in Thoracic Disease" was commissioned by the 
editorial office without any funding or sponsorship. EM has received honoraria from Astra Zeneca Pharmaceuticals, Merck \& Co, and received research support from Pfizer, Astra Zeneca, Merck, BMS, GSK, and Tessa Pharmaceuticals. The other authors have no other conflicts of interest to declare.

Ethical Statement: The authors are accountable for all aspects of the work in ensuring that questions related to the accuracy or integrity of any part of the work are appropriately investigated and resolved.

Open Access Statement: This is an Open Access article distributed in accordance with the Creative Commons Attribution-NonCommercial-NoDerivs 4.0 International License (CC BY-NC-ND 4.0), which permits the noncommercial replication and distribution of the article with the strict proviso that no changes or edits are made and the original work is properly cited (including links to both the formal publication through the relevant DOI and the license). See: https://creativecommons.org/licenses/by-nc-nd/4.0/.

\section{References}

1. Siegel RL, Miller KD, Jemal A. Cancer statistics, 2019. CA Cancer J Clin 2019;69:7-34.

2. Reck M, Rodríguez-Abreu D, Robinson AG, et al. Pembrolizumab versus Chemotherapy for PD-L1Positive Non-Small-Cell Lung Cancer. N Engl J Med 2016;375:1823-33.

3. Mok TSK, Wu YL, Kudaba I, et al. Pembrolizumab versus chemotherapy for previously untreated, PD-L1expressing, locally advanced or metastatic non-small-cell lung cancer (KEYNOTE-042): a randomised, open-label, controlled, phase 3 trial. Lancet 2019;393:1819-30.

4. Carbone DP, Reck M, Paz-Ares L, et al. First-Line Nivolumab in Stage IV or Recurrent Non-Small-Cell Lung Cancer. N Engl J Med 2017;376:2415-26.

5. Socinski MA, Jotte RM, Cappuzzo F, et al. Atezolizumab for First-Line Treatment of Metastatic Nonsquamous NSCLC. N Engl J Med 2018;378:2288-301.

6. Antonia SJ, Villegas A, Daniel D, et al. Durvalumab after Chemoradiotherapy in Stage III Non-Small-Cell Lung Cancer. N Engl J Med 2017;377:1919-29.

7. Postow MA, Sidlow R, Hellmann MD. Immune-Related Adverse Events Associated with Immune Checkpoint Blockade. N Engl J Med 2018;378:158-68.

8. Hellmann MD, Ciuleanu TE, Pluzanski A, et al. Nivolumab plus Ipilimumab in Lung Cancer with a High Tumor Mutational Burden. N Engl J Med 2018;378:2093-104.

9. Hellmann MD, Paz-Ares L, Bernabe Caro R, et al. Nivolumab plus Ipilimumab in Advanced Non-Small-Cell Lung Cancer. N Engl J Med 2019;381:2020-31.

10. Borghaei H, Paz-Ares L, Horn L, et al. Nivolumab versus Docetaxel in Advanced Nonsquamous Non-Small-Cell Lung Cancer. N Engl J Med 2015;373:1627-39.

11. Brahmer J, Reckamp KL, Baas P, et al. Nivolumab versus Docetaxel in Advanced Squamous-Cell Non-Small-Cell Lung Cancer. N Engl J Med 2015;373:123-35.

12. Herbst RS, Baas P, Kim DW, et al. Pembrolizumab versus docetaxel for previously treated, PD-L1-positive, advanced non-small-cell lung cancer (KEYNOTE-010): a randomised controlled trial. Lancet 2016;387:1540-50.

13. Rittmeyer A, Barlesi F, Waterkamp D, et al. Atezolizumab versus docetaxel in patients with previously treated non-small-cell lung cancer (OAK): a phase 3, openlabel, multicentre randomised controlled trial. Lancet 2017;389:255-65.

14. Antonia SJ, Borghaei H, Ramalingam SS, et al. Four-year survival with nivolumab in patients with previously treated advanced non-small-cell lung cancer: a pooled analysis. Lancet Oncol 2019;20:1395-408.

15. Ilie M, Khambata-Ford S, Copie-Bergman C, et al. Use of the 22C3 anti-PD-L1 antibody to determine PD-L1 expression in multiple automated immunohistochemistry platforms. PLoS One 2017;12:e0183023.

16. Yu H, Boyle TA, Zhou C, et al. PD-L1 Expression in Lung Cancer. J Thorac Oncol 2016;11:964-75.

17. Mu CY, Huang JA, Chen Y, et al. High expression of PD-L1 in lung cancer may contribute to poor prognosis and tumor cells immune escape through suppressing tumor infiltrating dendritic cells maturation. Med Oncol 2011;28:682-8.

18. Pawelczyk K, Piotrowska A, Ciesielska U, et al. Role of PD-L1 Expression in Non-Small Cell Lung Cancer and Their Prognostic Significance according to Clinicopathological Factors and Diagnostic Markers. Int J Mol Sci 2019;20:824.

19. Miyazawa T, Marushima H, Saji H, et al. PD-L1 Expression in Non-Small-Cell Lung Cancer Including Various Adenocarcinoma Subtypes. Ann Thorac Cardiovasc Surg 2019;25:1-9.

20. Goodman AM, Sokol ES, Frampton GM, et al. Microsatellite-Stable Tumors with High Mutational Burden Benefit from Immunotherapy. Cancer Immunol Res 2019;7:1570-3. 
21. Hellmann MD, Nathanson T, Rizvi H, et al. Genomic Features of Response to Combination Immunotherapy in Patients with Advanced Non-Small-Cell Lung Cancer. Cancer Cell 2018;33:843-52 e4.

22. Perea F, Sánchez-Palencia A, Gómez-Morales M, et al. HLA class I loss and PD-L1 expression in lung cancer: impact on T-cell infiltration and immune escape. Oncotarget 2017;9:4120-33.

23. Peng W, Liu C, Xu C, et al. PD-1 blockade enhances T-cell migration to tumors by elevating IFN-gamma inducible chemokines. Cancer Res 2012;72:5209-18.

24. Dulos J, Carven GJ, van Boxtel SJ, et al. PD-1 blockade augments Th1 and Th17 and suppresses Th2 responses in peripheral blood from patients with prostate and advanced melanoma cancer. J Immunother 2012;35:169-78.

25. Karachaliou N, Gonzalez-Cao M, Crespo G, et al. Interferon gamma, an important marker of response to immune checkpoint blockade in non-small cell lung cancer and melanoma patients. Ther Adv Med Oncol 2018;10:1758834017749748.

26. Higgs BW, Morehouse CA, Streicher K, et al. Interferon Gamma Messenger RNA Signature in Tumor Biopsies Predicts Outcomes in Patients with Non-Small Cell Lung Carcinoma or Urothelial Cancer Treated with Durvalumab. Clin Cancer Res 2018;24:3857-66.

27. Skoulidis F, Goldberg ME, Greenawalt DM, et al. STK11/LKB1 Mutations and PD-1 Inhibitor Resistance in KRAS-Mutant Lung Adenocarcinoma. Cancer Discov 2018;8:822-35.

28. Fujimoto D, Yoshioka H, Kataoka Y, et al. Pseudoprogression in Previously Treated Patients with Non-Small Cell Lung Cancer Who Received Nivolumab Monotherapy. J Thorac Oncol 2019;14:468-74.

29. Katz SI, Hammer M, Bagley SJ, et al. Radiologic Pseudoprogression during Anti-PD-1 Therapy for Advanced Non-Small Cell Lung Cancer. J Thorac Oncol 2018;13:978-86.

30. Kim HK, Heo MH, Lee HS, et al. Comparison of RECIST to immune-related response criteria in patients with non-small cell lung cancer treated with immunecheckpoint inhibitors. Cancer Chemother Pharmacol 2017;80:591-8.

31. Kawai O, Ishii G, Kubota K, et al. Predominant infiltration of macrophages and CD8(+) T Cells in cancer nests is a significant predictor of survival in stage IV non-small cell lung cancer. Cancer 2008;113:1387-95.

32. Stumpf $M$, Hasenburg A, Riener MO, et al. Intraepithelial CD8-positive $\mathrm{T}$ lymphocytes predict survival for patients with serous stage III ovarian carcinomas: relevance of clonal selection of T lymphocytes. Br J Cancer 2009;101:1513-21.

33. Seo JW, Tavaré R, Mahakian LM, et al. CD8+ T-Cell Density Imaging with 64Cu-Labeled Cys-Diabody Informs Immunotherapy Protocols. Clin Cancer Res 2018;24:4976-87.

34. Tavaré R, Escuin-Ordinas H, Mok S, et al. An Effective Immuno-PET Imaging Method to Monitor CD8Dependent Responses to Immunotherapy. Cancer Res 2016;76:73-82.

35. Sun R, Limkin EJ, Vakalopoulou M, et al. A radiomics approach to assess tumour-infiltrating CD8 cells and response to anti-PD-1 or anti-PD-L1 immunotherapy: an imaging biomarker, retrospective multicohort study. Lancet Oncol 2018;19:1180-91.

36. Chen DS, Mellman I. Elements of cancer immunity and the cancer-immune set point. Nature 2017;541:321-30.

37. Cesano A, Warren S. Bringing the Next Generation of Immuno-Oncology Biomarkers to the Clinic. Biomedicines 2018;6:14.

38. Hartimath SV, Draghiciu O, van de Wall S, et al. Noninvasive monitoring of cancer therapy induced activated T cells using [(18)F]FB-IL-2 PET imaging. Oncoimmunology 2016;6:e1248014.

39. Higashikawa K, Yagi K, Watanabe K, et al. 64Cu-DOTAanti-CTLA-4 mAb enabled PET visualization of CTLA4 on the T-cell infiltrating tumor tissues. PLoS One 2014;9:e109866.

40. Natarajan A, Mayer AT, Xu L, et al. Novel Radiotracer for ImmunoPET Imaging of PD-1 Checkpoint Expression on Tumor Infiltrating Lymphocytes. Bioconjug Chem 2015;26:2062-9.

41. Hettich M, Braun F, Bartholoma MD, et al. HighResolution PET Imaging with Therapeutic Antibodybased PD-1/PD-L1 Checkpoint Tracers. Theranostics 2016;6:1629-40.

42. England CG, Ehlerding EB, Hernandez R, et al. Preclinical Pharmacokinetics and Biodistribution Studies of $89 \mathrm{Zr}$-Labeled Pembrolizumab. J Nucl Med 2017;58:162-8.

43. Cole EL, Kim J, Donnelly DJ, et al. Radiosynthesis and preclinical PET evaluation of (89)Zr-nivolumab (BMS936558) in healthy non-human primates. Bioorg Med Chem 2017;25:5407-14.

44. England CG, Jiang D, Ehlerding EB, et al. (89)Zrlabeled nivolumab for imaging of $T$-cell infiltration in a humanized murine model of lung cancer. Eur J Nucl Med 
Mol Imaging 2018;45:110-20.

45. Broos K, Keyaerts M, Lecocq Q, et al. Non-invasive assessment of murine PD-L1 levels in syngeneic tumor models by nuclear imaging with nanobody tracers.

Oncotarget 2017;8:41932-46.

46. Severin GW, Engle JW, Barnhart TE, et al. 89Zr radiochemistry for positron emission tomography. Med Chem 2011;7:389-94.

47. Maude SL, Frey N, Shaw PA, et al. Chimeric antigen receptor $\mathrm{T}$ cells for sustained remissions in leukemia. $\mathrm{N}$ Engl J Med 2014;371:1507-17.

48. Neelapu SS, Locke FL, Bartlett NL, et al. Axicabtagene Ciloleucel CAR T-Cell Therapy in Refractory Large

Cite this article as: Pharaon R, Koczywas MA, Salgia S, Mohanty A, Massarelli E. Biomarkers in immunotherapy: literature review and future directions. J Thorac Dis 2020;12(9):5119-5127. doi: 10.21037/jtd.2020.04.15
B-Cell Lymphoma. N Engl J Med 2017;377:2531-44.

49. Brown CE, Alizadeh D, Starr R, et al. Regression of Glioblastoma after Chimeric Antigen Receptor T-Cell Therapy. N Engl J Med 2016;375:2561-9.

50. Bagley SJ, Desai AS, Linette GP, et al. CAR T-cell therapy for glioblastoma: recent clinical advances and future challenges. Neuro Oncol 2018;20:1429-38.

51. Minn I, Rowe SP, Pomper MG. Enhancing CAR T-cell therapy through cellular imaging and radiotherapy. Lancet Oncol 2019;20:e443-51.

52. Emami-Shahri N, Foster J, Kashani R, et al. Clinically compliant spatial and temporal imaging of chimeric antigen receptor T-cells. Nat Commun 2018;9:1081. 